Supporting information

\title{
Direct Growth of Ferroelectric Oxide Thin Films on Polymers Through Laser-Induced Low-Temperature Liquid-Phase Crystallization
}

Min Gyu Kang, Myoung-Sub Noh, Jung Joon Pyeon, Woo-Suk Jung, Hi Gyu Moon, SeungHyub Baek, Sahn Nahm, Seok-Jin Yoon, and Chong-Yun Kang*

*Corresponding author. Email: cykang@kist.re.kr

This file includes:

Section S1: Numerical simulation of laser-induced temperature distribution in LNO and PZT thin films

Section S2: Structural and electrical characteristics of the excimer laser annealed PZT and LNO films

Section S3: Self-powered piezoelectric sensors exploiting flexible PZT thin film

\section{Figures}

Figure S1. Modeling conditions.

Figure S2. Heat transfer simulation results (LNO thin film).

Figure S3. Heat-transfer simulation results (PZT thin film).

Figure S4. Surface morphologies of the amorphous PZT and crystalline PZT after a laser pulse irradiation of $60 \mathrm{~mJ} / \mathrm{cm}^{2}$ energy.

Figure S5. XRD patterns of the PZT thin film grown on a crystalline LNO layer through LLC under $60 \mathrm{~mJ} / \mathrm{cm}^{2}$ energy for different numbers of irradiated laser pulses.

Figure S6. XRD patterns of the PZT thin film grown through LLC on a crystalline LNO layer and without the LNO layer.

Figure S7. Structural and electrical properties of an excimer laser annealed LNO thin film.

Figure S8. EDS mapping analysis of the crystallized PZT/LNO grown on PI.

Figure S9. TEM image at low magnification and SAED pattern collected in the PZT region.

Figure S10. Surface morphologies of the flexible PZT thin films before and after 100 times bending with various magnifications. 
Figure S11. Fabrication process for a self-powered strain and acceleration sensor.

Figure S12. XRD pattern of the crystalline PZT thin film grown on a crystalline BTO layer by LLC.

Figure S13. (a) Voltage signal from the flexible PZT sensor with and without poling. (b) Experimental setup for measuring the strain and acceleration rate of the sensor under periodic bending-releasing motion.

Figure S14. Schematic for the calculation of surface strain of the sensor.

\section{Tables}

Table S1. Material parameters for heat transfer modeling.

\section{Other supplementary information}

Movie S1. Self-powered flexible piezoelectric sensor. 


\section{Section S1: Numerical simulation of laser-induced temperature distribution in LNO and PZT thin films}

\section{Heat transfer simulation}

In this study, the homogenized excimer laser beam was used as a primary heat source to achieve low-temperature crystallization of the complex oxide thin film. The intense excimer laser energy dramatically increases the surface temperature of the thin film. However, unfortunately, the exact surface temperature and temperature distribution profile across the film is difficult to quantify through the experimental measurement because the excimer laser has an extremely short pulse width $(\sim 24 \mathrm{~ns})$. In this study, to calculate the temperature distribution profiles in the $\mathrm{PbZr}_{0.52} \mathrm{Ti}_{0.48} \mathrm{O}_{3}$ (PZT) and $\mathrm{LaNiO}_{3}$ (LNO) thin films, numerical modeling was performed using the modified heat-transfer equation. The heat-transfer equation in solids generally given by, ${ }^{1}$

$$
\rho C_{p} \frac{\partial T}{\partial t}-\nabla\left(k_{t} \nabla T\right)=G t(y, t)
$$

where, $\rho, C_{\mathrm{p}}, T, t, k_{\mathrm{t}}$, and $G t(y, t)$ are the material density, specific heat capacity, temperature, time, thermal conductivity, and heat source, respectively. The excimer laser energy is absorbed on the surface of the films, and the generated heat is transferred along the depth $(y)$ direction. According to Beer-Lambert law, therefore, the laser-driven heat source can be described by the equation:

$$
G t(y, t)=I(t) \cdot(1-R(T)) \frac{e^{\frac{-y}{\delta_{a}}}}{\delta_{a}}
$$

where, $I(\mathrm{t}), R(T)$, and $\delta_{\mathrm{a}}$ are the time distribution of the laser beam intensity at the surface, reflectivity, and penetration depth, respectively. Typically, the time dependence of the energy distribution of the excimer laser exhibits a Gaussian shape. Therefore, the laser intensity $I(t)$ can be defined as,

$$
I(\mathrm{t})=\frac{E}{(S \cdot \tau)} e^{-4 \frac{(t-\tau)^{2}}{\tau^{2}}}
$$

where, $E, S$, and $\tau$ are the applied laser energy, surface area, and pulse width, respectively. The reflectivity $R(T)$ in Eq. (S2) can be calculated from the complex index of refraction $(\tilde{\mathrm{n}})=\mathrm{n}+\mathrm{ik}$, where, $\mathrm{n}$ and $\mathrm{k}$ are the refractive index and extinction coefficient, respectively. The relationship between reflectivity and complex index of refraction is defined as,

$$
R(\mathrm{~T})=\frac{(n-1)^{2}+k^{2}}{(n+1)^{2}+k^{2}}
$$

The penetration depth in Eq. S2 can be obtained from the extinction coefficient:

$$
\delta_{\alpha}=\frac{1}{\alpha}=\frac{c}{2 w k}=\frac{4 \pi k}{\lambda}
$$


where, $\alpha, \omega, c$, and $\lambda$ are the optical absorption coefficient, angular frequency, speed of light, and laser wavelength, respectively. From the Eq. (S1), the time-dependent temperature distribution profile of the oxide thin films was calculated in this study.

The numerical simulation was performed using the COMSOL multi-physics software with the heat-transfer module. The results were plotted as temperature distribution profiles versus time as a function of depths $(y)$ and various laser energy density. The material parameters such as density $\rho$, heat capacity $C_{\mathrm{p}}$, and thermal conductivity $k_{\mathrm{t}}$ were obtained from literature. ${ }^{1-3}$ To calculate the reflectivity and penetration depth of the films, the actual $n$ and $k$ values of the amorphous PZT and LNO thin films at $248 \mathrm{~nm}$ wavelength were measured by ellipsometry. The values of the material parameters are summarized in Table S1.

Table S1. Material parameters for heat transfer modeling. ${ }^{1-3}$

\begin{tabular}{|l|l|l|}
\hline Parameters & PZT & LNO \\
\hline$n$ & 2.284 & 1.658 \\
\hline$k$ & 0.792 & 0.688 \\
\hline$R(T)$ & 0.2 & 0.12 \\
\hline$\delta_{\alpha}[\mathrm{nm}]$ & 24.91 & 28.68 \\
\hline$\rho\left[\mathrm{kg} / \mathrm{m}^{3}\right]$ & 8100 & 7200 \\
\hline$C_{p}[\mathrm{~J} /(\mathrm{kgK})]$ & 364 & 494 \\
\hline$k_{t}[\mathrm{~W} /(\mathrm{mK})]$ & 1.52 & 15 \\
\hline
\end{tabular}

\section{Modeling conditions}

For the modeling, a $125 \mu \mathrm{m}$-thick polyimide (PI) film was selected as the substrate, and $\mathrm{SiO}_{2}$ $(500 \mathrm{~nm})$ and $\mathrm{Si}_{3} \mathrm{~N}_{4}(500 \mathrm{~nm})$ were used as thermal insulation layers to prevent the thermal deformation of the PI films. A $300 \mathrm{~nm}$-thick LNO layer was formed on the $\mathrm{SiO}_{2}$ layer, as shown in Figure S1a. For the LNO film, laser energies were selected in a range from 50 to $70 \mathrm{~mJ} / \mathrm{cm}^{2}$. The ambient temperature was chosen as $200{ }^{\circ} \mathrm{C}$. The surface boundary condition for the laserirradiated area can be defined as,

$$
\vec{n} \cdot(k \nabla T)=q_{0}+h\left(T_{\infty}-T\right)+\sigma \cdot \epsilon\left(T_{a m b}^{4}-T^{4}\right)
$$

where, $\vec{n}, q_{0}, h, T_{\text {inf }}, \sigma, \varepsilon$, and $T_{\mathrm{amb}}$ are the normal vector, surface heat flux, convective heat transfer coefficient, external temperature, Stefan-Boltzmann constant, emissivity, and ambient 
temperature, respectively. The other boundaries are assumed to be thermally insulated (adiabatic conditions), given by the equation,

$$
\vec{n} \cdot(k \nabla T)=0
$$

For the PZT film formed on the LNO layer, the thickness varied from $50 \mathrm{~nm}$ to $150 \mathrm{~nm}$. The irradiated laser energy was varied from 40 to $60 \mathrm{~mJ} / \mathrm{cm}^{2}$ (Figure S1b).
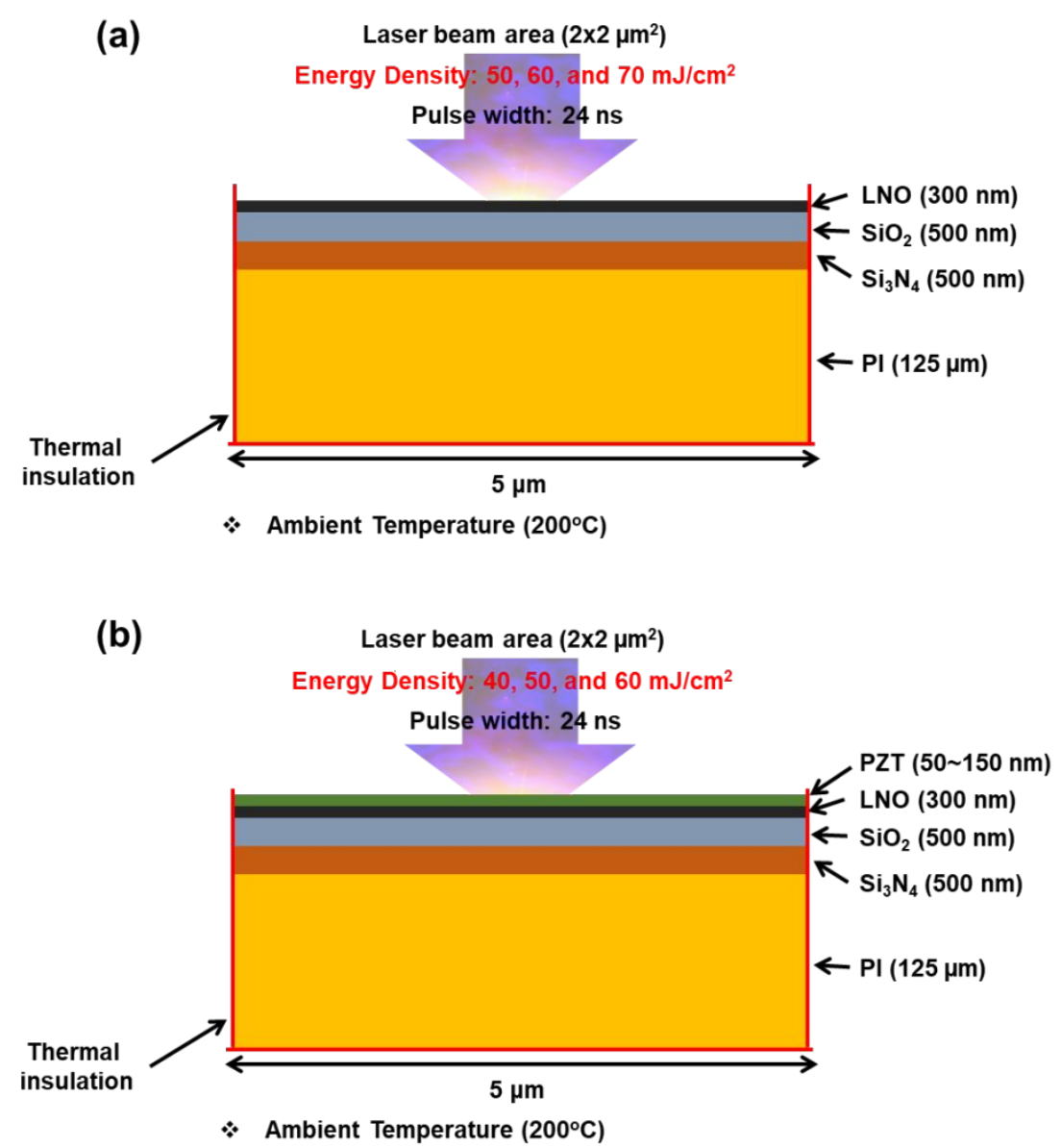

Figure S1. Modeling conditions (a) for the LNO thin film and (a) PZT thin film.

\section{Simulation results}

Figure S2 shows the temperature profiles of the $300 \mathrm{~nm}$-thick LNO thin film. The surface temperature is increased with increasing irradiated laser energy, as shown in Figure S2a-c. The temperature at the surface and $\mathrm{LNO} / \mathrm{SiO}_{2}$ interface show the difference, because most of the applied laser energy is absorbed at the surface, and the heat energy is diffused into the interior of the LNO thin film. The temperature difference between the surface and $\mathrm{LNO} / \mathrm{SiO}_{2}$ interface is increased with increasing applied laser energy, as shown in Figure S2d-f. 
(a)

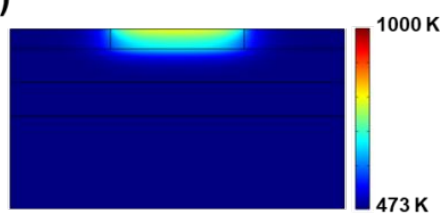

(d)

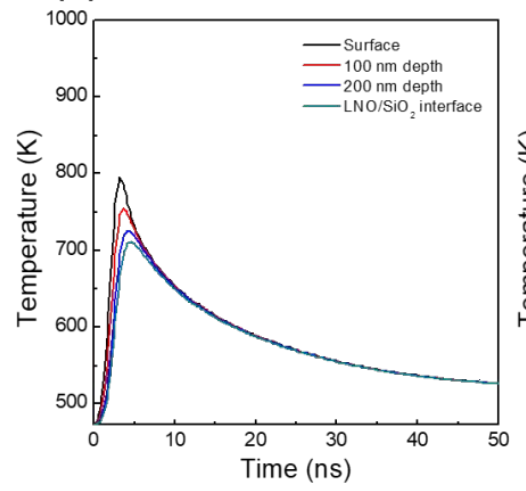

(b)

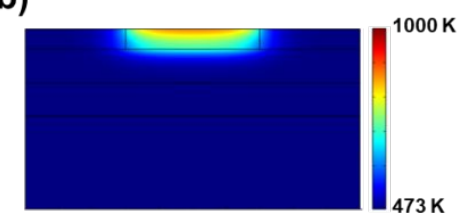

(e)

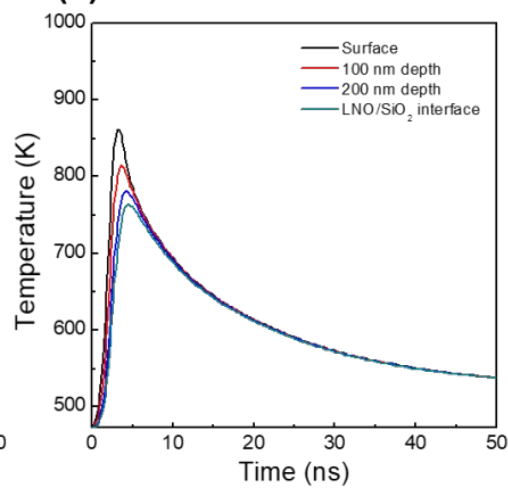

(c)

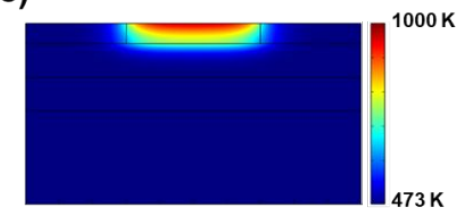

(f)

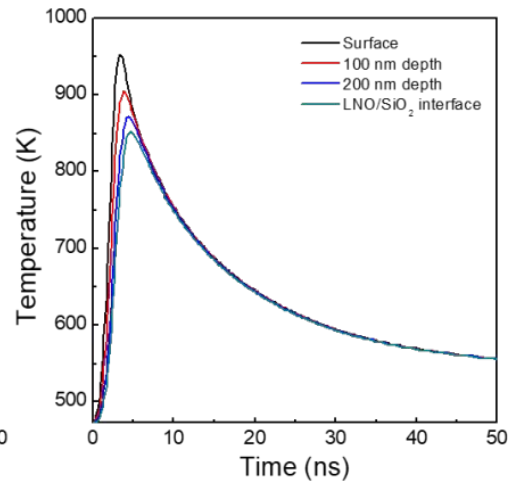

Figure S2. Heat transfer simulation results (LNO thin film). Temperature distribution after laser irradiation under (a) $50 \mathrm{~mJ} / \mathrm{cm}^{2}$ (b) $60 \mathrm{~mJ} / \mathrm{cm}^{2}$ (c) $70 \mathrm{~mJ} / \mathrm{cm}^{2}$. Time dependence of the temperature profiles as a function of depth after laser irradiation under (d) $50 \mathrm{~mJ} / \mathrm{cm}^{2}$ (e) 60 $\mathrm{mJ} / \mathrm{cm}^{2}$ (f) $70 \mathrm{~mJ} / \mathrm{cm}^{2}$.

Figure S2 shows the temperature profiles for the PZT thin film. The surface temperature of the PZT thin film is much higher compared to that of the LNO film under the same laser energy. Moreover, the temperature difference between the surface and PZT/LNO interface is much more significant than that of the LNO film. As a result, it can be revealed that the absorbed thermal energy is accumulated in the surface region because of the low thermal conductivity of the PZT, which is about 10 times lower than that of the LNO. This behavior becomes significant with the increasing thickness of the PZT film. Figure S3a-c show the temperature distribution profiles of the PZT thin film after laser irradiation under $40 \mathrm{~mJ} / \mathrm{cm}^{2}$ with different thickness. Although the applied laser energy is the same, the surface temperature is sharply increased, and the temperature difference between the surface and PZT/LNO interface is increased depending on the film thickness. This result indicates that the LNO thin film, which has a higher thermal conductivity, dissipates heat from the PZT layer, and this plays a role in avoiding the accumulation of thermal energy in the surface region. The influence of the LNO film is significantly reduced when the thickness of the PZT layer is increased. As a result, it is revealed that synthesizing fully crystallized PZT thin film by the excimer laser is challenging owing to the significant temperature difference between the surface and PZT/LNO interface. For example, the surface temperature of the 150 -nm thick PZT film is raised to $\sim 1450 \mathrm{~K}$, which leads to thermal damage in the film, when $60 \mathrm{~mJ} / \mathrm{cm}^{2}$ laser energy is supplied to the surface, whereas the temperature at the PZT/LNO interface is only $\sim 700 \mathrm{~K}$, which is too low to crystallize PZT film. 

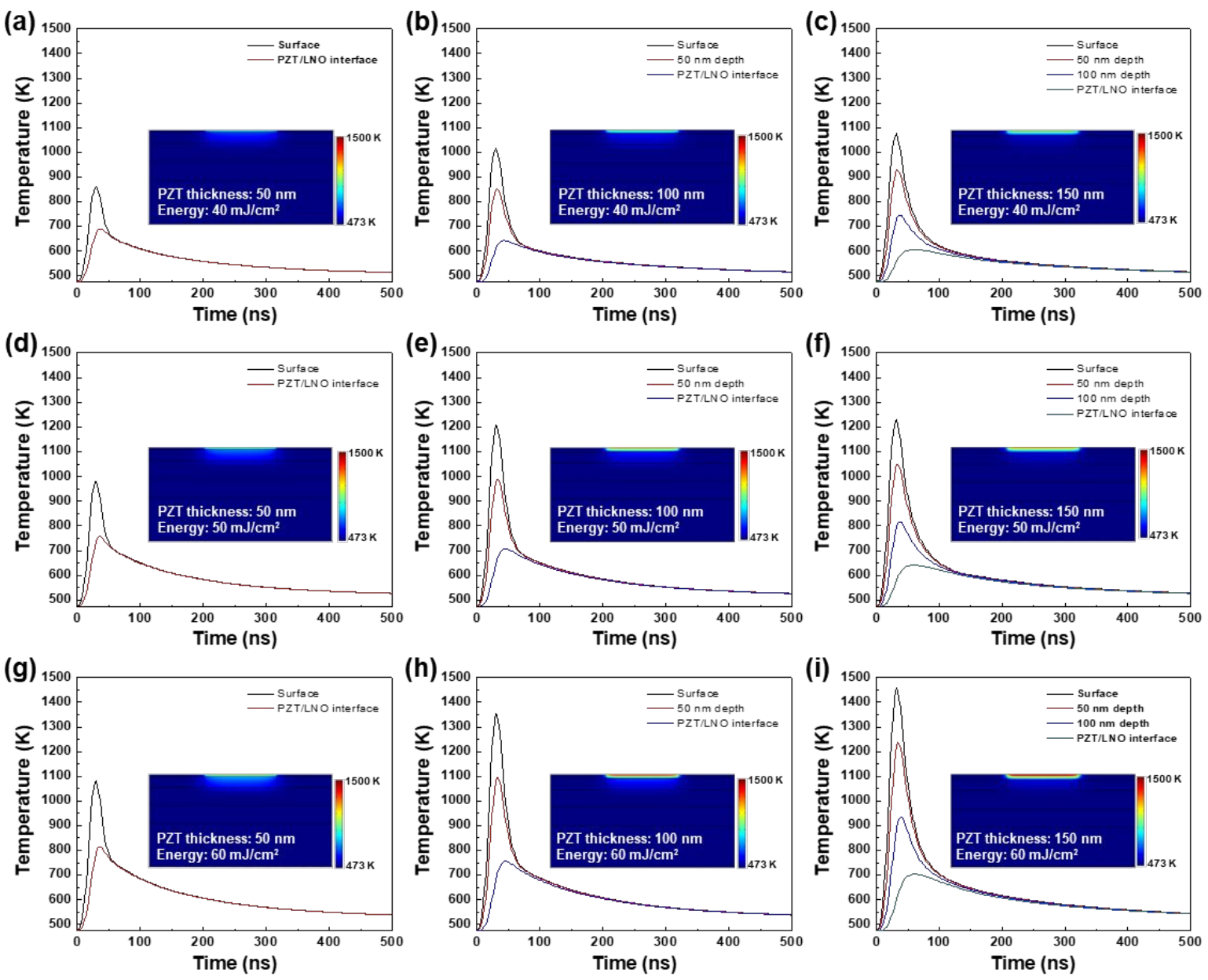

Figure S3. Heat-transfer simulation results (PZT thin film). Time dependence of temperature profiles as a function of depth after laser irradiation under $40 \mathrm{~mJ} / \mathrm{cm}^{2}$ for different thicknesses: (a) $50 \mathrm{~nm}$, (b) $100 \mathrm{~nm}$, and (c) $150 \mathrm{~nm}$. Time dependence of temperature profiles as a function of depth after laser irradiation under $50 \mathrm{~mJ} / \mathrm{cm}^{2}$ for different thicknesses: (d) $50 \mathrm{~nm}$, (e) 100 $\mathrm{nm}$, and (f) $150 \mathrm{~nm}$. Time dependence of temperature profiles as a function of depth after laser irradiation under $60 \mathrm{~mJ} / \mathrm{cm}^{2}$ for different thicknesses: (g) $50 \mathrm{~nm}$, (h) $100 \mathrm{~nm}$, and (i) $150 \mathrm{~nm}$. 
Section S2: Structural and electrical characteristics of the excimer laser annealed PZT and LNO films
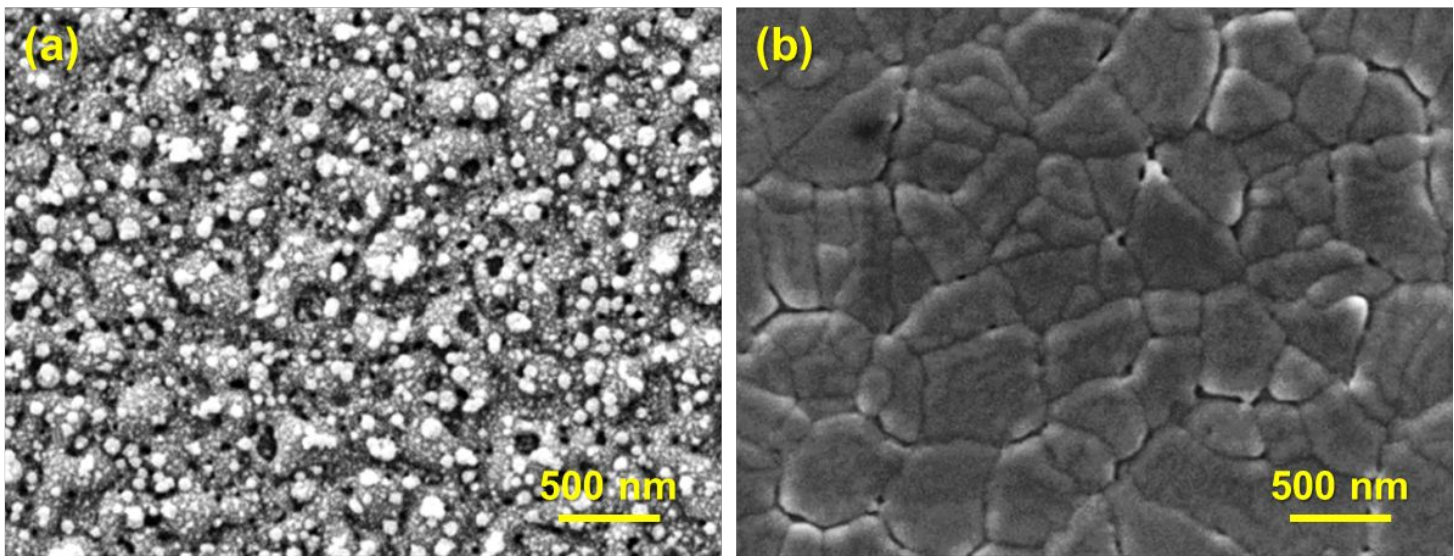

Figure S4. Surface morphologies of (a) the amorphous PZT and (b) crystalline PZT after a laser pulse irradiation of $60 \mathrm{~mJ} / \mathrm{cm}^{2}$ energy. The crystalline PZT thin film was synthesized by high temperature heat treatment at $650{ }^{\circ} \mathrm{C}$.

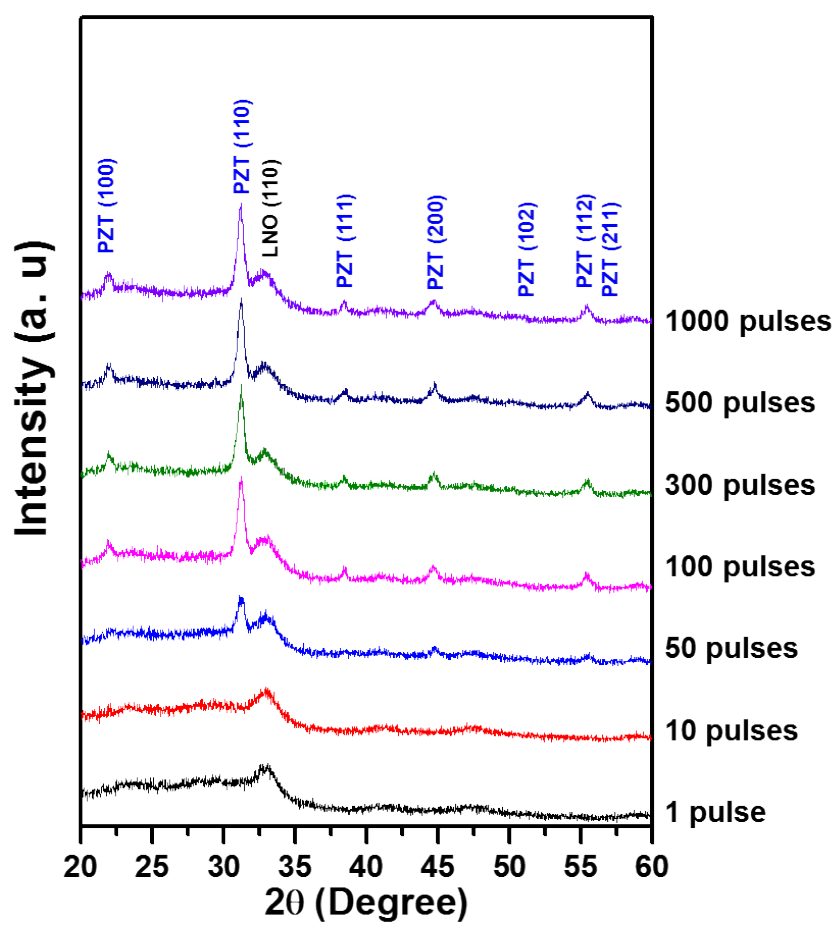

Figure S5. XRD patterns of the PZT thin film grown on a crystalline LNO layer through LLC under $60 \mathrm{~mJ} / \mathrm{cm}^{2}$ energy for different number of irradiated laser pulses. 


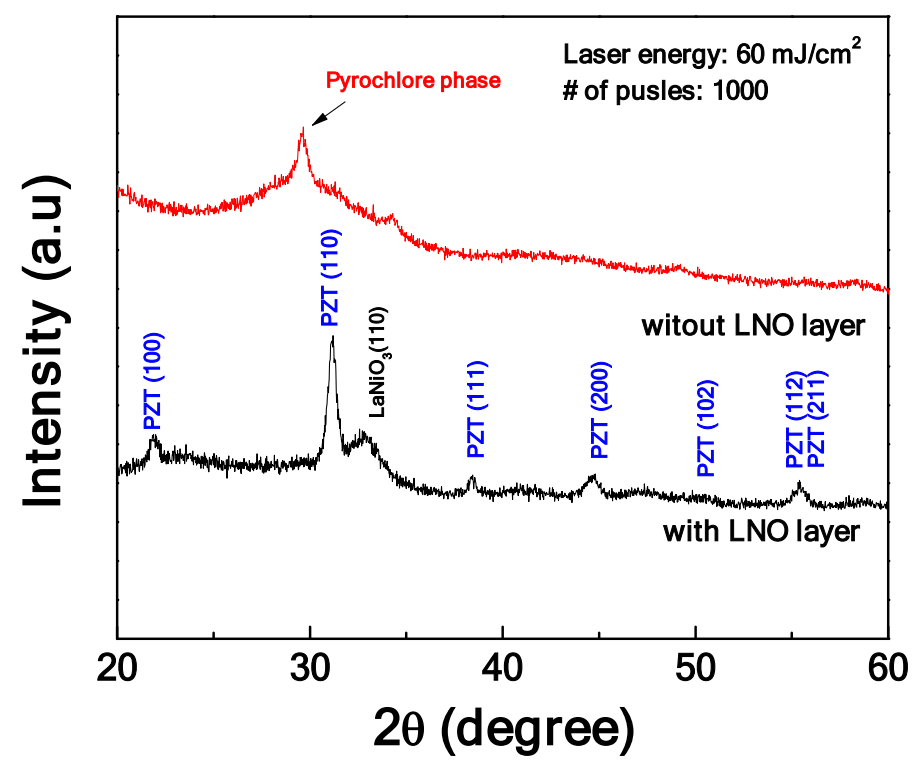

Figure S6. XRD patterns of the PZT thin film grown through LLC on a crystalline LNO layer (bottom line) and without the LNO layer (upper line).

(a)

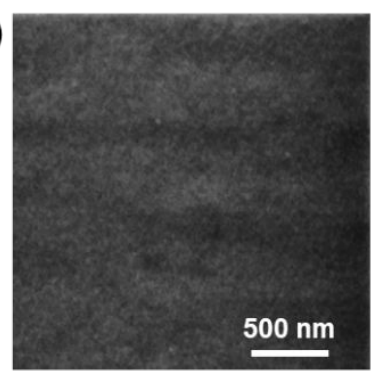

(b)

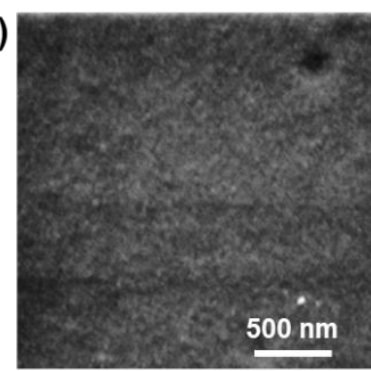

(d)

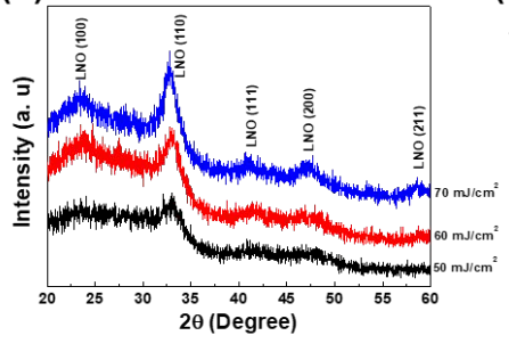

$(\mathrm{e})_{10}$

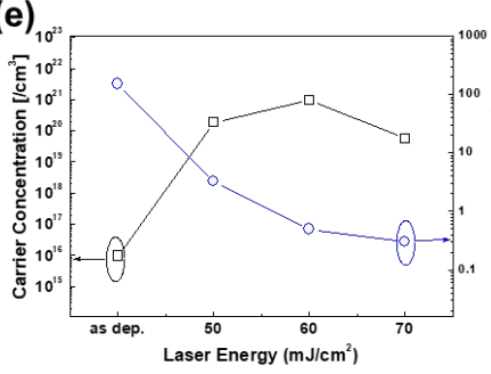

(c)

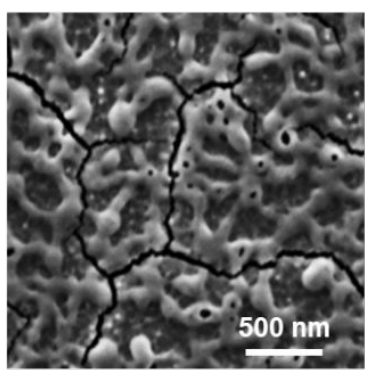

(f)

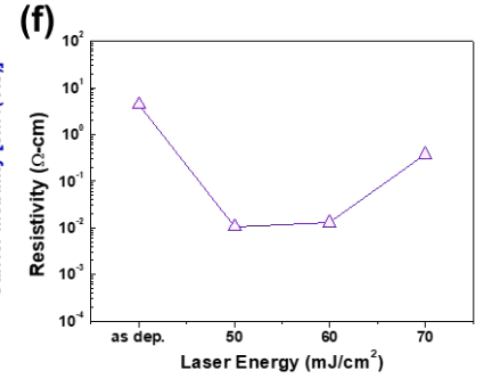

Figure S7. Structural and electrical properties of an excimer laser annealed LNO thin film. Surface morphologies of the crystallized LNO thin film under (a) $50 \mathrm{~mJ} / \mathrm{cm}^{2}$ (b) $60 \mathrm{~mJ} / \mathrm{cm}^{2}$ (c) $70 \mathrm{~mJ} / \mathrm{cm}^{2}$. (d) XRD patterns of the LNO thin films for different laser energy. (e) Carrier concentration and mobility of the LNO thin film for different laser energy. (f) Resistivity of the LNO thin films for different laser energy. 


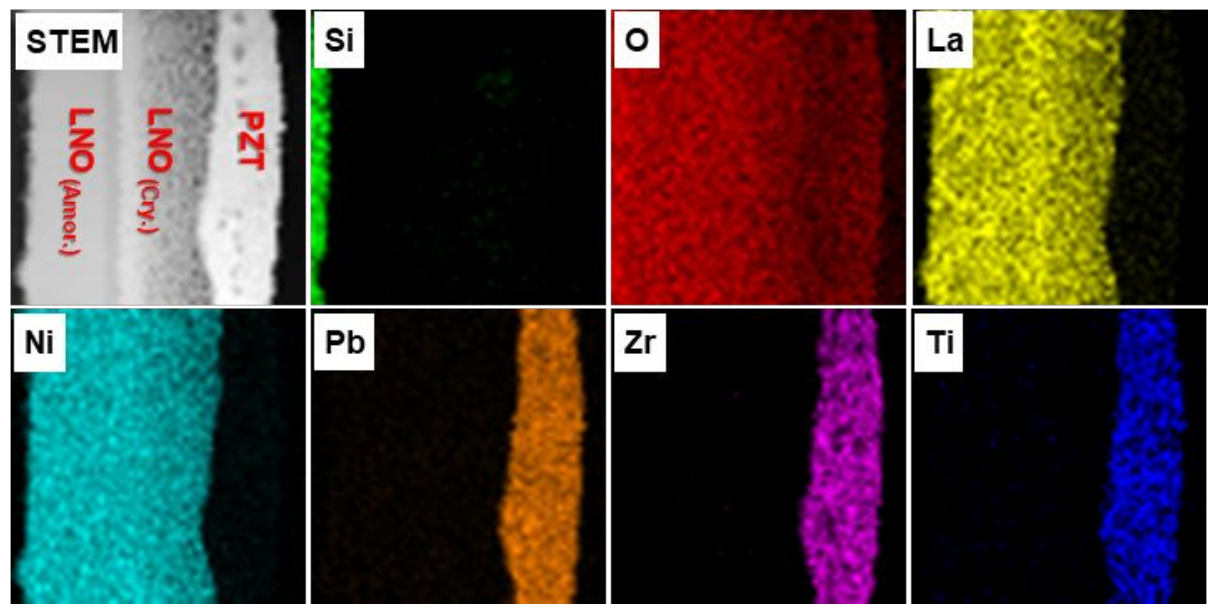

Fig. S8. EDS mapping analysis of the crystallized PZT/LNO grown on PI.

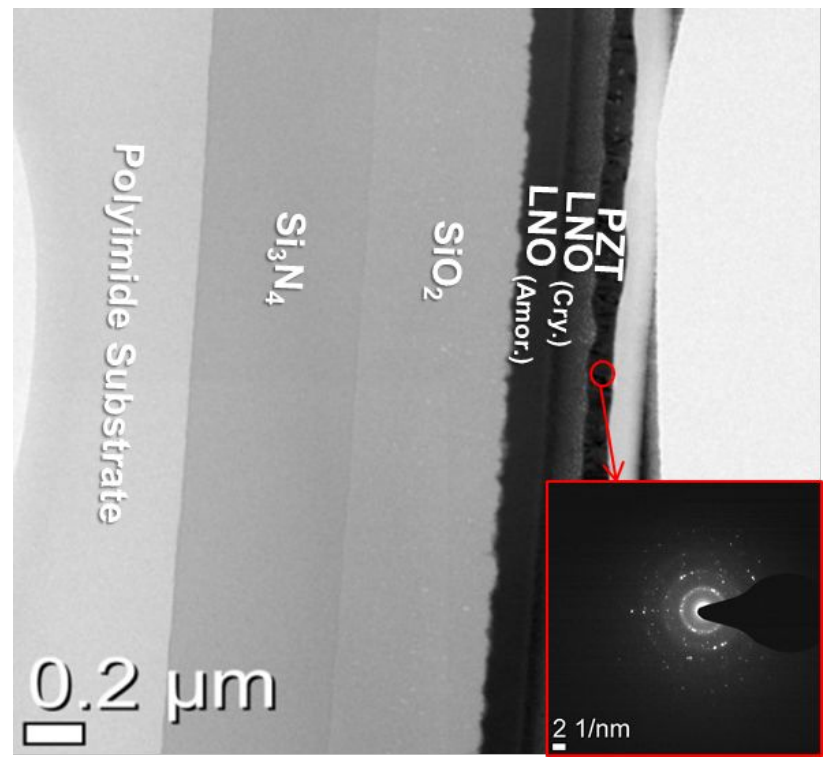

Figure S9. TEM image at a low magnification. (inset) SAED pattern collected in the PZT region. 


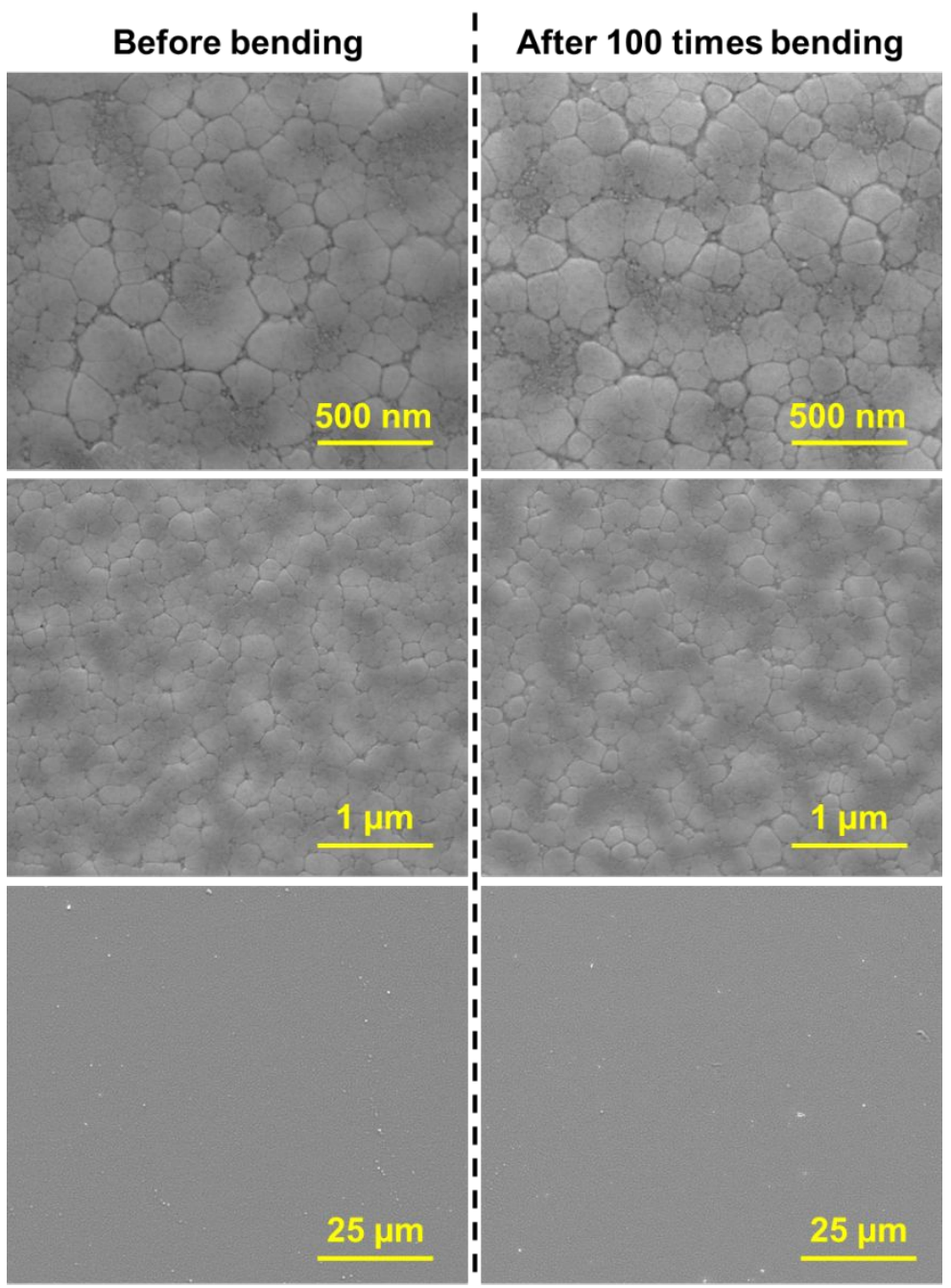

Figure S10. Surface morphologies of the flexible PZT thin films before and after 100 times bending with various magnifications. 
(a)
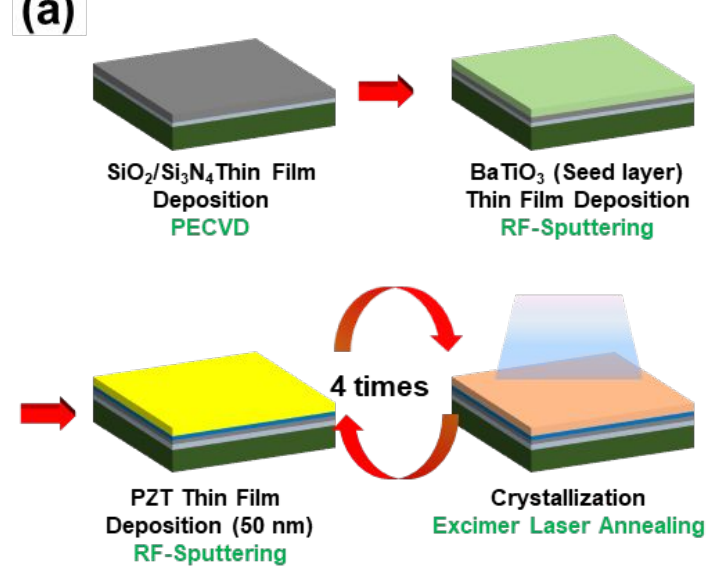
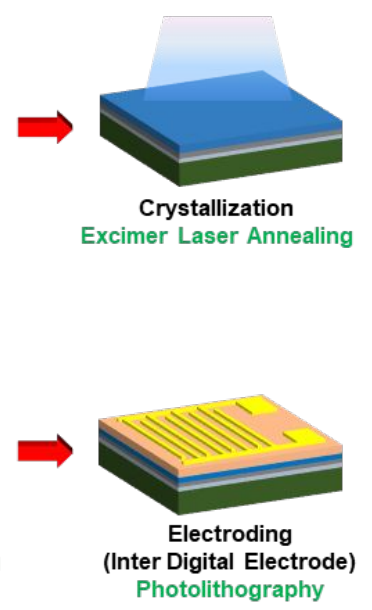

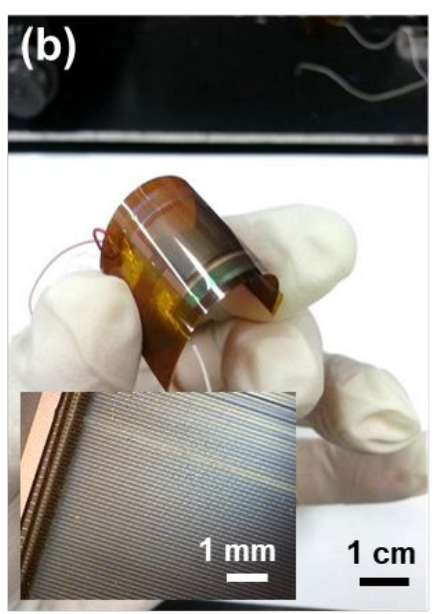

Figure S11. Fabrication process for the self-powered strain and acceleration sensor. (a) Process flow to fabricate a self-powered strain and acceleration sensor. (b) Photograph of the self-powered piezoelectric sensor. The inset represents an optical microscopy image of the IDE pattern.

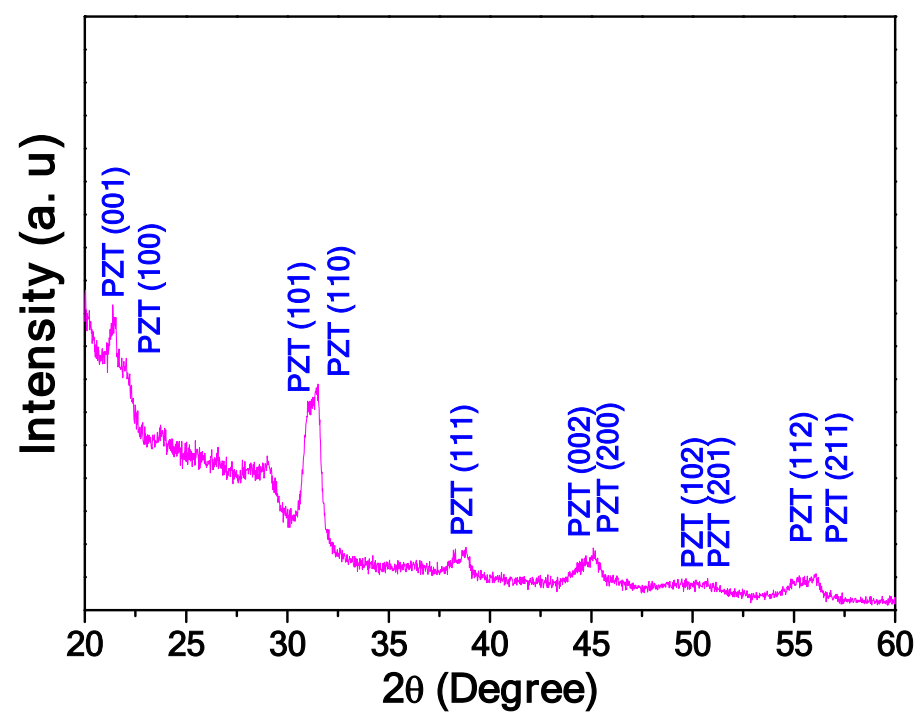

Figure S12. XRD pattern of the crystalline PZT thin film grown on a crystalline BTO layer by LLC. 

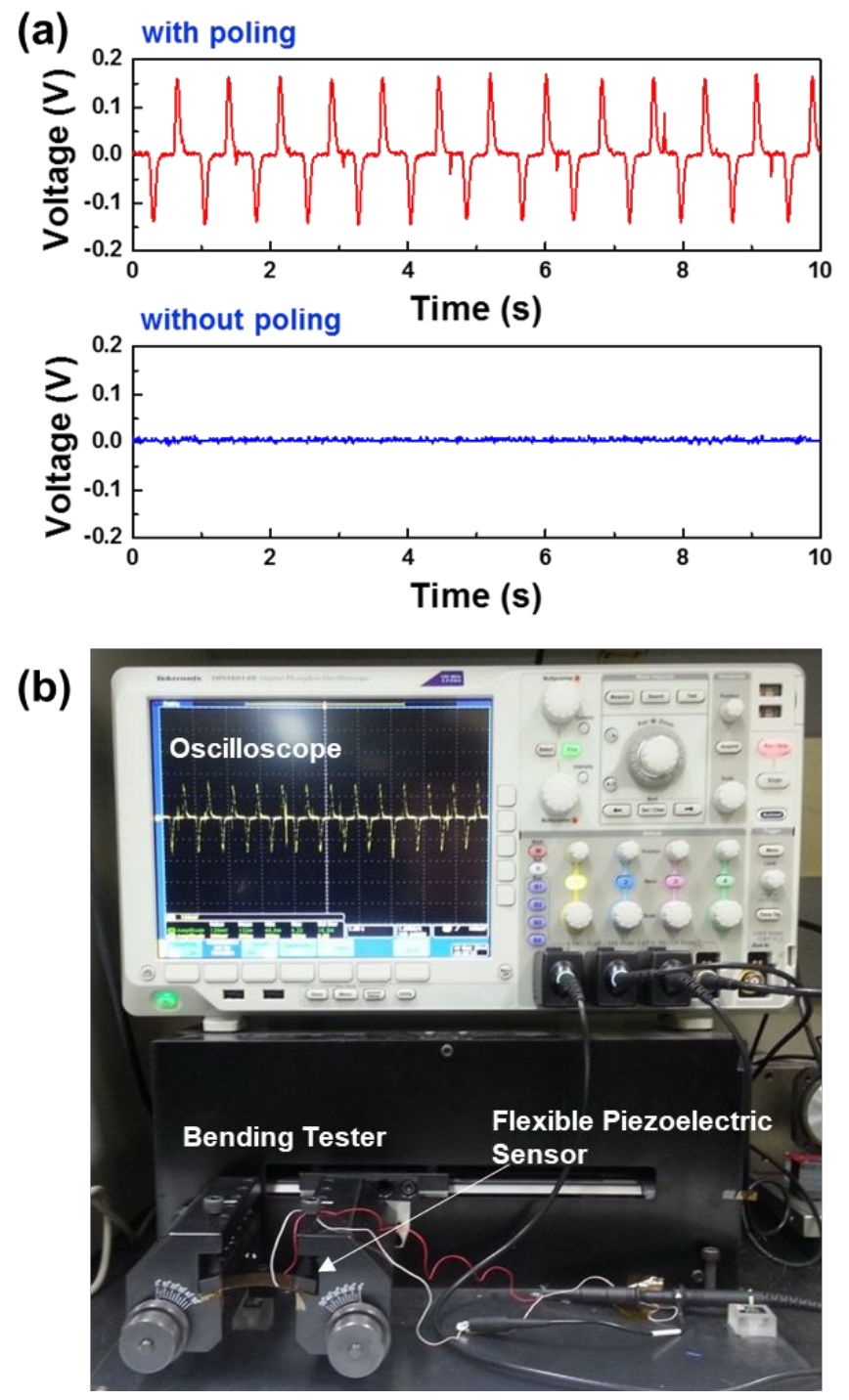

Figure S13. (a) Voltage signal from the flexible PZT sensor with and without poling. (b) Experimental setup for measuring the strain and acceleration rate of the sensor under periodic bending-releasing motion. 


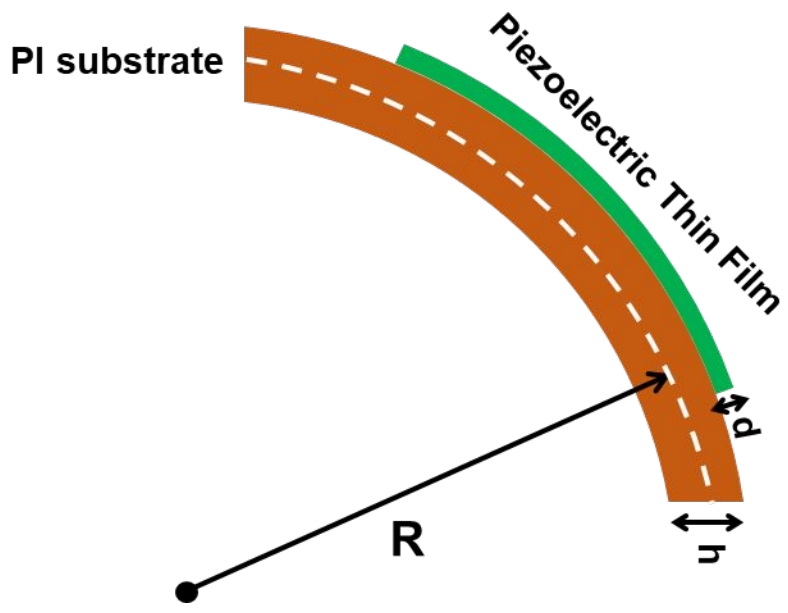

Figure S14. Schematic for the calculation of surface strain of the sensor.

The surface strain of the PI substrate was calculated using the following equation,

$$
\epsilon=\frac{h}{2 R}
$$

where $\varepsilon, h$, and $R$ are the strain, thickness of the PI substrate, and bending radius. The PZT film has a very thin thickness, $d$. Because $R>>h$ and $h>>d$, the strain in the PZT thin film is approximately equal to the strain on the outer surface of the PI substrate (Fig. S14).

\section{References}

1. Donohue, P. P.; Todd, M. A., Pulse-extended excimer laser annealing of lead zirconate titanate thin films. Integr. Ferroelectr. 2000, 31 (1-4), 285-296.

2. Zinkevich, M.; Solak, N.; Nitsche, H.; Ahrens, M.; Aldinger, F., Stability and thermodynamic functions of lanthanum nickelates. Journal of Alloys and Compounds 2007, 438 (1), 92-99.

3. Lai, S. C.; Lue, H.-T.; Hsieh, K. Y.; Lung, S. L.; Liu, R.; Wu, T. B.; Donohue, P. P.; Rumsby, P., Extended-pulse excimer laser annealing of $\mathrm{Pb}(\mathrm{Zr1} 1-\mathrm{xTix}) \mathrm{O} 3$ thin film on LaNiO3 electrode. Journal of Applied Physics 2004, 96 (5), 2779-2784. 